\title{
Branched Silver Nanowires as Controllable Plasmon Routers
}

\author{
Yurui Fang, ${ }^{\dagger}$ Zhipeng Li, ${ }^{\dagger}$ Yingzhou Huang, ${ }^{\dagger}$ Shunping Zhang, ${ }^{\dagger}$ Peter Nordlander, ${ }^{\ddagger}{ }^{\dagger}$ \\ Naomi J. Halas, ${ }^{\mp, \dagger}$ and Hongxing $\mathrm{Xu}^{\dagger, \S, *}$
}

${ }^{\dagger}$ Beijing National Laboratory for Condensed Matter Physics and Institute of Physics, Chinese Academy of Sciences,
Box 603-146, 100190 , Beijing, China, ${ }^{*}$ Department of Physics and Astronomy, Department of Electrical and
Computer Engineering, Laboratory for Nanophotonics, Rice University, Houston, Texas 77005 , and ${ }^{\S}$ Division of
Solid State Physics/The Nanometer Consortium, Lund University, Box 118, S-22100, Lund, Sweden

ABSTRACT Using polarization dependent scattering spectroscopy, we investigate plasmon propagation on branched silver nanowires. By controlling the polarization of the incident laser light, the wire plasmons can be routed into different wire branches and result in light emission from the corresponding wire ends. This routing behavior is found to be strongly dependent on the wavelength of light. Thus for certain incident polarizations, light of different wavelength will be routed into different branches. The branched nanowire can thus serve as a controllable router and multiplexer in integrated plasmonic circuits.

KEYWORDS Router, plasmon propagation, polarization, branched silver nanowire, switch, plasmonic circuit

S urface plasmons provide a channel for the nanoscale manipulation of light and enable a wide range of applications ranging from ultrasensing,,${ }^{1-4}$ superlensing, ${ }^{5,6}$ nanolasing, ${ }^{7,8}$ cloaking ${ }^{9,10}$ to photothermal cancer therapies. ${ }^{11,12}$ The significant recent progress in plasmonics has stimulated scientists to develop nanoscale plasmonic analogues of macro- and microscale optical components such as electrooptic devices, ${ }^{13,14}$ waveguides, ${ }^{15-23}$ and modulators. ${ }^{24,25}$ By combining nanoscale optical devices it may be possible to build integrated nanophotonic circuits, offering substantial improvements in bandwidth and speed for next-generation information technologies. ${ }^{26,27}$ As a central component of this future technology, plasmon waveguiding has been studied extensively, including light-plasmon in/out-coupling, ${ }^{19,20,28}$ plasmon propagation, ${ }^{21,29}$ and splitting. ${ }^{30,31}$ However, the key functional elements of nanophotonic circuits such as plasmon routers, multiplexers, switchers, and logic gates have not yet been realized. Here we show that branched silver nanowire structures can serve as controllable plasmonic routers and multiplexers.

The crystalline Ag nanowires were fabricated using a selfseeding progress, yielding Ag nanowires with a mean diameter of $\sim 150 \mathrm{~nm}$, and lengths ranging from one to tens of micrometers. ${ }^{32}$ Ethanolic suspension of Ag nanowires was then spin-coated on ITO glass slides patterned with indexed grids and dried under ambient conditions. With the help of the indexed grids, each nanowire can be clearly identified in both optical and SEM images. Occasionally, branched nanowire structures were formed. The sample is immersed in the oil immediately after the SEM measurement to avoid

* To whom correspondence should be addressed. E-mail: hxxu@iphy.ac.cn. Received for review: 04/3/2010

Published on Web: 04/26/2010 the oxidation of Ag nanowires in air. The plasmon propagations on the branched nanowire structures are then investigated by an optical microscope system as illustrated in Figure 1. The light from the 632.8 and/or $785 \mathrm{~nm}$ lasers are introduced through a $100 \times$ oil immerged objective to excite one end of the nanowires. Emission signals are recorded by a CCD (DVC-1412AM high-resolution digital camera) or a spectrometer, which are selectable by the position of mirror 3. The laser polarization is controlled by a half-wave plate.

Several examples of branched Ag nanowire structures that function as plasmonic routers are shown in Figure 2. As evident in the high resolution SEM images, the gaps separating the branches are a few tens of nanometers. When one end of the wires is excited with a $633 \mathrm{~nm}$ laser, surface plasmon polaritons (SPPs) are launched and propagate along

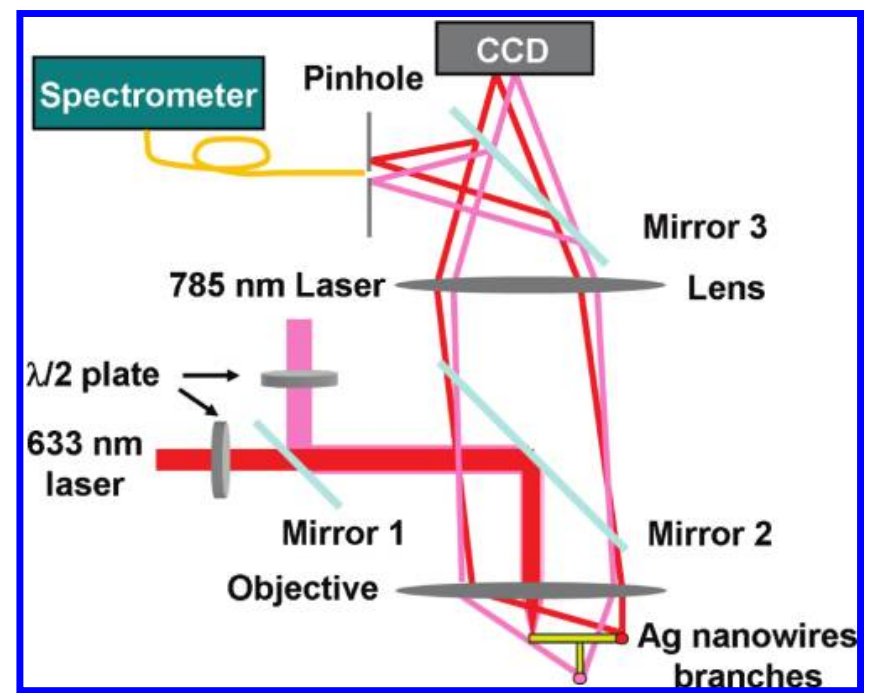

FIGURE 1. Schematic illustration of the experimental setup. 


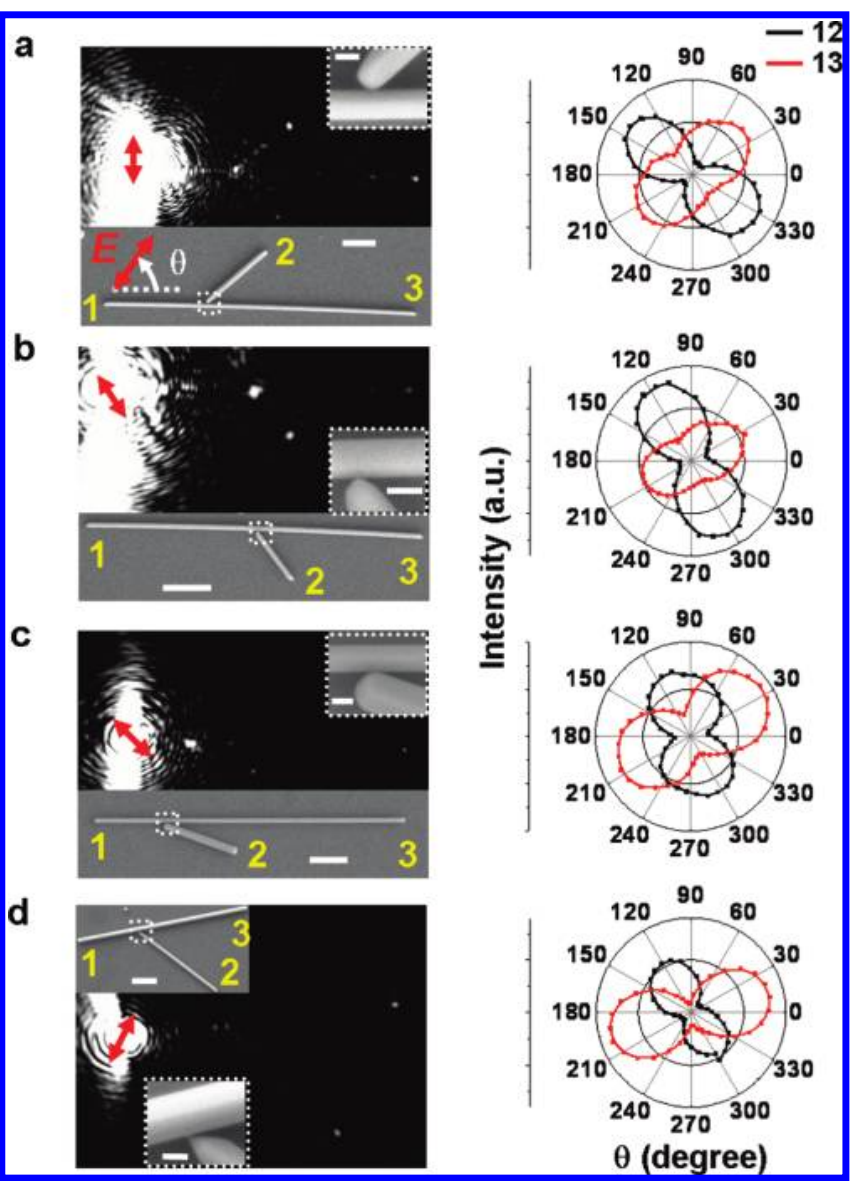

FIGURE 2. (Left column) Optical images of the light emitted at the ends of nanowires from plasmons launched by exciting the branched wire structure at wire end 1 with $633 \mathrm{~nm}$ laser light. Red arrows represent the polarization orientation of the excitation laser. (Insets) Corresponding SEM images of the router structures and the junctions, where the scale bars are all $2 \mu \mathrm{m}$ and $200 \mathrm{~nm}$, respectively. $\theta$ is the incident polarization angle. (Right column) Polar plots of the emission intensity from wire ends 2 (black) and 3 (red) as a function of polarization angle $\theta$ for the corresponding structure shown on the left.

the wires. It is clear that SPPs cannot only propagate to the other end of the main wire, but also couple to and propagate along the branch wires. Branched Ag nanowire structures can thus function as passive plasmonic beam splitters. ${ }^{22,23}$ However, we find in these structures that the relative intensities of SPPs can be directed to either the main or the branch wire by changing the polarization of the excitation light, as shown in the polar plots of the intensities of the light emission from the different ends of each structure. For example, the relative intensities of light emitted from the wire ends 2 and 3 in Figure 2 a depend strongly on incident polarization. For an incident polarization of $140^{\circ}$, strong light emission is achieved at wire end 2 , while light emission at wire end 3 is very dim. The plasmon propagation is thus almost completely switched to the branch wire for this incident polarization. For an incident polarization of $40^{\circ}$, the situation is reversed. The emission intensity at the wire end 3 is large while the emission at the wire end 2 is minimal.

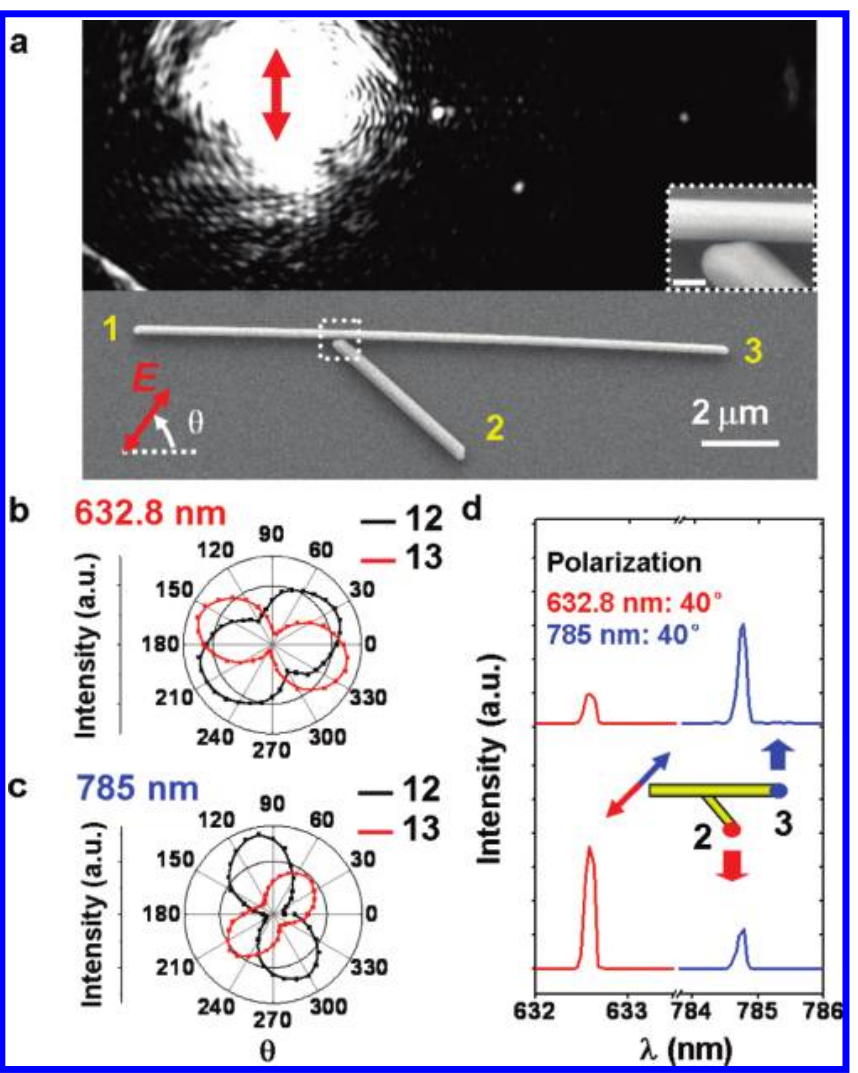

FIGURE 3. (a) Optical image of a silver nanobranch excited by a 633 $\mathrm{nm}$ wavelength laser. The red arrow represents the incident polarization. The inset is the SEM image of the nanobranch and the junction, where the scale bars are $2 \mu \mathrm{m}$ and $200 \mathrm{~nm}$, respectively. $\theta$ is the rotation angle of the incident polarization. (b,c) Emission intensity from branch ends 2 (black) and 3 (red) as a function of incident polarization angle for 633 and $785 \mathrm{~nm}$ wavelength excitation, respectively. (d) The spectra collected from wire end 2 (upper curves) and 3 (lower curves). The polarization of the incident light is $40^{\circ}$.

For this polarization, the surface plasmons launched by the incident light remain in the main wire and is not diverted into the branch. The other examples of branched nanowire structures (Figure 2b-d and Figure S1 in Supporting Information) show similar behavior, although in each case different incident polarizations are required to selectively route the light onto either channel of the nanostructure. We believe that it is a coincidence that the routers shown in Figure 2 have similar angles for the minima and maxima of their 12 and 13 amplitudes (Data from the wires discussed in Figure 3 and S1 in Supporting Information, as well as from several other routers not presented, show different angles for the routing). However, in all cases shown, when emission from the end of the main wire is at a minimum, emission from the end of the side wire becomes maximal, and vice versa. In this way, propagating SPPs in this structure can be specifically routed to distinct nanowire destinations by controlling the incident polarization. The routing of the light is very efficient. The maximum switching ratio $I_{2} / I_{3}$ or $I_{3} / I_{2}$ at the optimal polarizations can reach as high as 8 . 
Branched nanowires also selectively route SPPs launched with distinct free-space wavelengths to different locations of the structure. A typical example of two-wavelength discrimination is shown in Figure 3. For excitation using the $633 \mathrm{~nm}$ laser, the maximum emission intensity from wire ends 2 and 3 are obtained for an incident polarization of 30 and $160^{\circ}$, respectively (Figure 3b). For SPPs launched at this free-space wavelength, we observe that some crosstalk between the main and branch SPP is present, although the routing behavior is still clearly observable. For $785 \mathrm{~nm}$ light, the nanostructure functions as an almost perfect plasmonic router, switching the SPPs between the main and branch wires at incidence polarizations of $\sim 45$ and $110^{\circ}$ very cleanly (Figure 3c). Interestingly, when this nanostructure is excited by two lasers of different wavelengths, the different wavelengths are routed differently. For certain polarizations, the light can be fully demultiplexed so that light of different wavelengths are emitted at different wire ends. For example as shown in Figure 3d, when 633 and $785 \mathrm{~nm}$ light is mixed and focused on wire end 1 with a polarization of $40^{\circ}$, the $633 \mathrm{~nm}$ light will be almost completely routed to the branch wire while the $785 \mathrm{~nm}$ light will be primarily routed in the main wire. When the incident polarization of one laser is fixed and the incident polarization of a second laser at a different wavelength is varied, the switching and routing behaviors are changed only for the light whose incident polarization is being varied (data not shown). Hence, the switching and routing functions of SPPs for the branched nanowire structures can work simultaneously and independently for multiple wavelengths without interfering, although the switching and routing behaviors may be different for different wavelengths.

From a detailed theoretical analysis of the routing behavior of smaller branched wire systems, a simple, clear picture of the microscopic mechanism of the observed routing dependence on the incident polarization has emerged. In a recent publication discussing the polarization properties of light emitted from individual nanowires, ${ }^{33}$ we show that for an arbitrary incident polarization, a superposition of wire plasmons of different wavelengths and azimuthal symmetries is generated. Coupling to the azimuthally symmetric $(m=0)$ wire plasmon is maximal for incident polarization parallel to the wire. Coupling to the azimuthally antisymmetric $(m=1)$ wire plasmon is maximal for incident polarization perpendicular to the wire. The interference of these wire plasmons modulates the near field distribution along the main wire. Since coupling to the different wire plasmons is polarization dependent, the near field distribution along the wire will depend on the input polarization. This is shown in Figure 4, where a theoretical analysis of the routing behavior in a typical branched nanowire using the Finite Element Method (Comsol) is shown. Panel a shows maximal emission from the branch for $\theta=27^{\circ}$ and minimal emission at $117^{\circ}$. Panel $b$ shows that for an incident polarization of $\theta=27^{\circ}$, the near field around the main wire

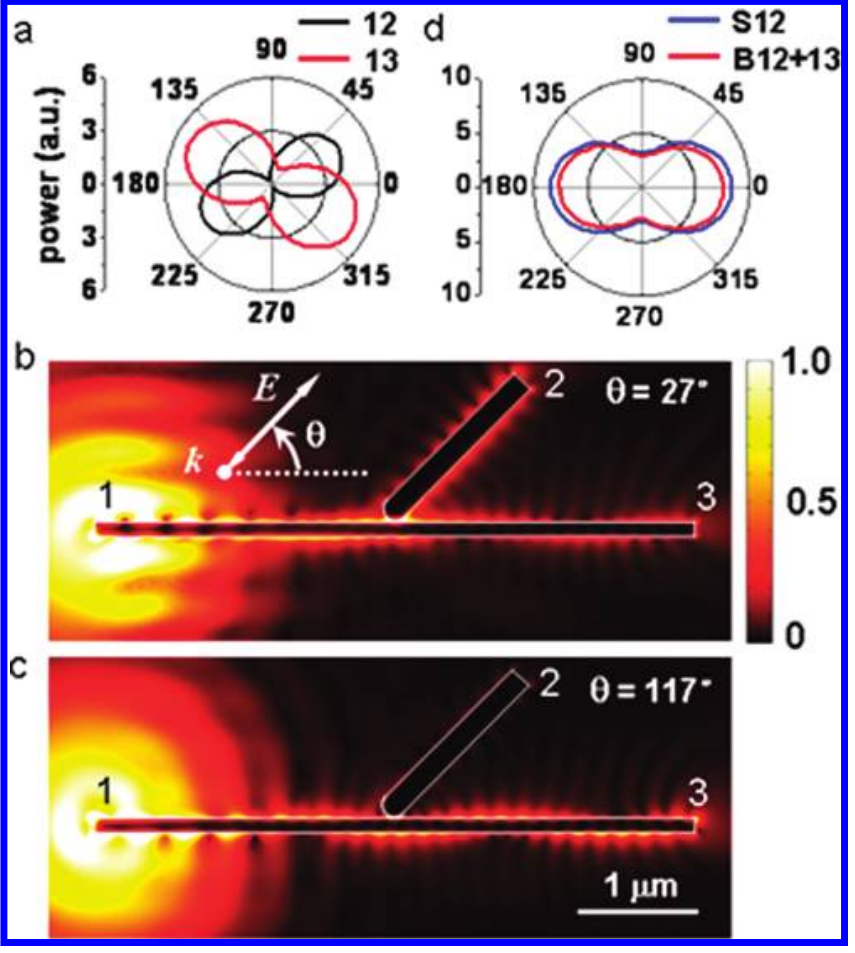

FIGURE 4. FEM simulations of silver nanowire branch excited by Gaussian beam (beam waist $1 \mu \mathrm{m}$ ). The coupling end of the branch wire $\left(L_{\mathrm{b}}=1.5 \mu \mathrm{m}, D_{\mathrm{b}}=200 \mathrm{~nm}\right)$ was capped by a hemisphere with a $10 \mathrm{~nm}$ gap from the main wire $\left(L_{\mathrm{m}}=5 \mu \mathrm{m}, D_{\mathrm{m}}=120 \mathrm{~nm}\right)$. (a) Emission power at terminals of main (red) and branch wire (black) as a function of incident polarization angle $\theta$ (indicated in panel b). Emission from the branch wire is maximal for $\theta=27^{\circ}$ and minimal at $\theta=117^{\circ}$. (b,c) Local electric field $|E|$ distributions at the maxima (b) and minima (c) emission at the branch wire. The maximal field enhancement was truncated at 1.0 to better show the near field along the nanowires. (d) Emission power S12 (blue) from a single wire of same dimension as the main wire, compared to the total emission power B12 +13 (red) from both terminals in the branched structure.

is very large at the branch junction. Thus light can couple efficiently into the branch. For an incident polarization of $117^{\circ}$, the near field in the junction is very small and almost no plasmons are excited in the branch and hence no emission as shown in panel c. In panel d, we compare the polarization dependence of the sum of the light emitted from both ends of the branched nanowire with the light emitted from the end of main trunk in the absence of the branch. The emission pattern is almost identical, showing that the presence of the branch does not influence the overall incoupling of light into the structure, but allows for an efficient routing of plasmons into the branch with very little loss. In Figure S3 in the Supporting Information, we show the experimental data for the emission characteristics of an individual nanowire. This data is in excellent agreement with the results in Figure $4 \mathrm{~d}$ and shows that the maximum coupling of light into a nanowire occurs for polarization parallel to the nanowire. ${ }^{33}$ The specific polarization characteristic of a branched nanowire structure depends on the shape of the main-branch junction, that is, the gap size and the geometric structure of the nearby branch wire end, and 


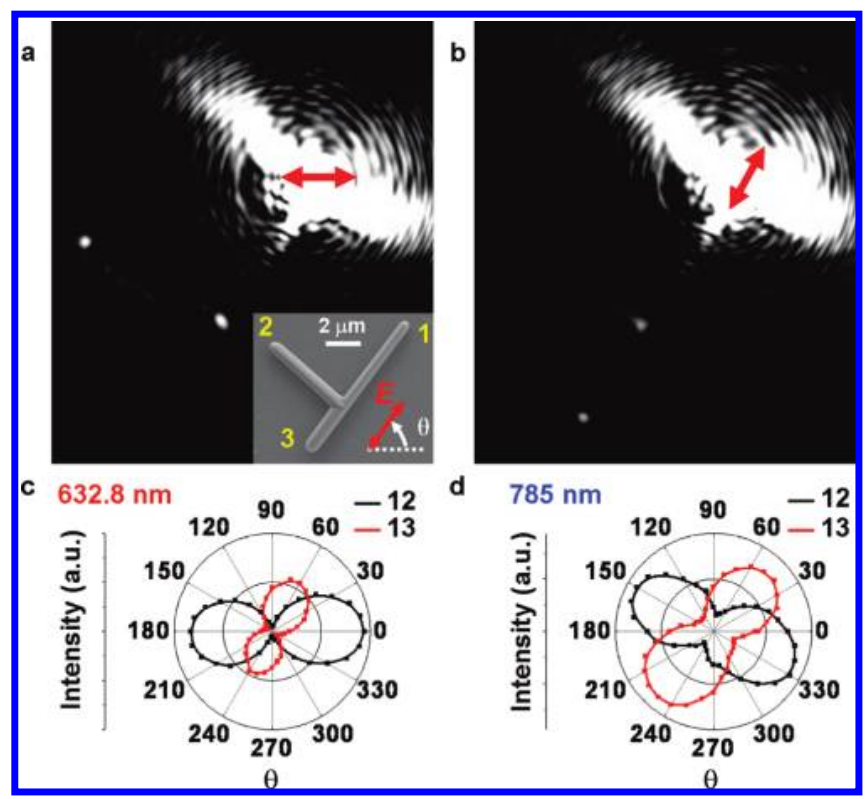

FIGURE 5. (a,b) Optical image of a silver nanobranch excited at wire end 1 by $633 \mathrm{~nm}$ laser light at different polarizations. The red arrow represents the incident polarization. The inset in (a) is the SEM image of the nanobranch. $\theta$ is the incident polarization angle. (c) Emission intensity from wire ends 2 (black) and 3 (red) of the branch for different incident polarization at $633 \mathrm{~nm}$ wavelength. (d) As for c but for $785 \mathrm{~nm}$ wavelength excitation.

also on the shape of the wire tip at the excitation end. A more detailed theoretical investigation of the routing characteristics of single and multibranched nanowires is in progress.

Occasionally, " $T$ " shaped nanowire structures can result from the nanowire synthesis method, as shown in the inset of Figure $5 \mathrm{a}$. For these structures, the plasmons can propagate directly from the main to the branch wire without evanescent coupling across the nanogap, as seen in the previous structures (Figures 2 and 3). In these continuously coupled wires, the plasmon propagation and routing efficiency is higher, indicating that the presence of the gap in previous structures reduces the coupling efficiency. As shown in the optical images in Figure 5a,b, plasmon routing from the branch wire to the main wire can be clearly observed when the incident polarization of the $633 \mathrm{~nm}$ light is changed from 0 to nominally $60^{\circ}$. Figure $5 \mathrm{c}$ shows the emission intensities from wire ends 2 and 3 as a function of incident polarization angle. The emission intensity from both 2 and 3 shows a highly regular $\cos ^{2} \theta$ dependence, which also suggests that a very efficient SPP routing can be achieved when the main and branch wires are continuously coupled. The switching ratio $I_{2} / I_{3}$ is about 13 at the incident polarization $\sim 90^{\circ}$, and $I_{3} / I_{2}$ is about 12 at the incident polarization $\sim 160^{\circ}$, which is larger than for the gap-coupled nanobranches in Figure 2. A similar routing behavior is also observed for $785 \mathrm{~nm}$ incident wavelength (Figure $5 \mathrm{~d}$ ). The case of excitation at the wire end 2 or 3 is shown in Figure S2 in Supporting Information.

In conclusion, we show that a branched nanowire structure can serve as a controllable, addressable plasmonic router and multiplexer. By changing the polarization of incident light, SPP propagation can be switched between the main and branch wires. The routing functions can work simultaneously for multiple wavelengths. The specific routing characteristics, as well as the intrinsic loss, are strongly affected by the specific geometry of the interwire junction. This easily controllable and addressable routing and multiplexing of light at the nanoscale may find numerous applications in future nanophotonic devices, circuits, and networks.

Acknowledgment. We thank Dr. Javier García de Abajo for valuable discussions. This work was supported by NSFC Grant 10625418, 10874233, 10904171 , MOST Grant 2006DFB02020, 2009CB930700, "Bairen Project" of CAS, the National Science Foundation under Grant CNS-0421109, the Robert A. Welch Foundation under Grants C-1220 and C-1222, and the Center for Advanced Solar Photophysics, an Energy Frontier Research Center funded by the U.S. Department of Energy, Office of Science, Office of Basic Energy Sciences.

Supporting Information Available. More examples of branched nanowire structures, "T" shaped nanowire with the excitation at (2) and (3). This material is available free of charge via the Internet at http://pubs.acs.org.

\section{REFERENCES AND NOTES}

(1) Li, Z. Y.; Xia, Y. N. Nano Lett. 2010, 10, 243-249.

(2) Zhang, Z.; Weber-Bargioni, A.; Wu, S. W.; Dhuey, S.; Cabrini, S.; Schuck, P. I. Nano Lett. 2009, 9, 4505-4509.

(3) Fang, Y. R.; Wei, H.; Hao, F.; Nordlander, P.; Xu, H. X. Nano Lett. 2009, 9, 2049-2053.

(4) Xu, H. X.; Bjerneld, E. J.; Käll, M.; Börjesson, L. Phvs. Rev. Lett 1999, 83, 4357-4360.

(5) Smolyaninov, I. I.; Hung, Y. J.; Christopher, C. D. Science 2007, 315, 1699-1700.

(6) Liu, Z. W.; Lee, H.; Xiong, Y.; Sun, C.; Zhang, X. Science 2007, 315, 1686-1686

(7) Noginov, M. A.; Zhu, G.; Belgrave, A. M.; Bakker, R.; Shalaev, V. M.; Narimanov, E. E.; Stout, S.; Herz, E.; Suteewong, T.; Wiesner, U. Nature 2009, 460, $1110-U 1168$.

(8) Oulton, R. F.; Sorger, V. J.; Zentgraf, T.; Ma, R. M.; Gladden, C.; Dai, L.; Bartal, G.; Zhang, X. Nature 2009, 461, 629-632.

(9) Fang, N.; Lee, H.; Sun, C.; Zhang, X. Science 2005, 308, 534-537.

(10) Alù, A.; Engheta, N. Phvs. Rev. Lett. 2008, 100, 113901.

(11) Loo, C.; Lowery, A.; Halas, N. J.; West, J.; Drezek, R. Nano Lett. 2005, 5, 709-711.

(12) Lal, S.; Clare, S. E.; Halas, N. I. Acc. Chem. Res. 2008, 41, $1842-$ 1851 .

(13) Neutens, P.; Van Dorpe, P.; De Vlaminck, I.; Lagae, L.; Borghs, G. Nat. Photonics 2009, 3, 283-286.

(14) Cai, W. S.; White, J. S.; Brongersma, M. L. Nano Lett. 2009, 9, 4403-4411

(15) Maier, S. A.; Kik, P. G.; Atwater, H. A.; Meltzer, S.; Harel, E.; Koel, B. E.; Requicha, A. G. Nat. Mater. 2003, 2, 229-232.

(16) Li, Z. P.; Hao, F.; Huang, Y. Z.; Fang, Y. R.; Nordlander, P.; Xu, H. X. Nano Lett. 2009, 9, 4383-4386.

(17) Ditlbacher, H.; Hohenau, A.; Wagner, D.; Kreibig, U.; Rogers, M.; Hofer, F.; Aussenegg, F. R.; Krenn, J. R. Phys. Rev. Lett. 2005, 95 , 257403.

(18) Sanders, A. W.; Routenberg, D. A.; Wiley, B. J.; Xia, Y. N.; Dufresne, E. R.; Reed, M. A. Nano Lett. 2006, 6, 1822-1826.

(19) Akimov, A. V.; Mukherjee, A.; Yu, C. L.; Chang, D. E.; Zibrov, A. S.; Hemmer, P. R.; Park, H.; Lukin, M. D. Nature 2007, 450, 402-406.

(20) Knight, M. W.; Grady, N. K.; Bardhan, R.; Hao, F.; Nordlander, P.; Halas, N. I. Nano Lett. 2007, 7, 2346-2350. 
(21) Manjavacas, A.; García de Abajo, F. I. Nano Lett. 2009, 9, 1285 1289.

(22) Yan, R. X.; Pausauskie, P.; Huang, J. X.; Yang, P. D. Proc. Natl. Acad. Sci. U.S.A. 2009, 106, 21045-21050.

(23) Guo, X.; Qiu, M.; Bao, J. M.; Wiley, B. J.; Yang, Q.; Zhang, X. N. Ma, Y. G.; Yu, H. K.; Tong, L. M. Nano Lett. 2009, 9, 4515-4519.

(24) Pala, R. A.; Shimizu, K. T.; Melosh, N. A.; Brongersma, M. L. Nano Lett. 2008, 8, 1506-1510.

(25) Zheng, Y. B.; Yang, Y. W.; Jensen, L.; Fang, L.; Juluri, B. K.; Flood, A. H.; Weiss, P. S.; Stoddart, J. F.; Huang, T. L. Nano Lett. 2009, 9, 819-825.

(26) Barnes, W. L.; Dereux, A.; Ebbesen, T. W. Nature 2003, 424, 824 830
(27) Zia, R.; Schuller, J. A.; Chandran, A.; Brongersma, M. L. Mater. Todar 2006, 9, 20-27

(28) Wei, H.; Ratchford, D.; Li, X. Q.; Xu, H. X.; Shih, C. K. Nano Lett. 2009, 9, 4168-4171.

(29) Jones, A. C.; Olmon, R. L.; Skrabalak, S. E.; Wiley, B. J.; Xia, Y. N. N.; Raschke, M. B. Nano Lett. 2009, 9, 2553-2558.

(30) Evlyukhin, A. B.; Bozhevolnyi, S. I.; Stepanov, A. L.; Krenn, J. R. Appl. Phys. B. 2006, 84, 29-34.

(31) Tai, C.; Chang, S. H.; Chiu, T. L. Opt. Soc. Am. B 2008, 25, $1387-$ 1392.

(32) Sun, Y. G.; Xia, Y. N. Adv. Mater. 2002, 14, 833-837.

(33) Li, Z. P.; Bao, K.; Fang, Y. Huang, Y.; Nordlander, P.; Xu, H. X. Nano Lett. [Online early release]. DOI:10.1021/nl100528c. 